\title{
Primitive Ontology and the Structure of Fundamental Physical Theories
}

Forthcoming in: D. Albert, A. Ney (eds.), "The Wave Function,” Oxford: Oxford University Press.

\author{
Valia Allori \\ Department of Philosophy, Northern Illinois University, \\ Zulauf Hall 920, Dekalb Il 60115 USA. \\ E-mail: vallori@niu.edu.
}

For a long time, it was believed that it was impossible to be realist about quantum mechanics. It took quite a while for the researchers in the foundations of physics, beginning with John Stuart Bell (1987), to convince others that such an alleged impossibility had no foundation. These days there are several quantum theories that can be interpreted in a realist fashion, among which Bohmian mechanics, the Ghirardi-Rimini-Weber (GRW) theory, and the many-worlds theory.

The debate, though, is far from being over. In what respect should we be realist regarding these theories? At least two different proposals have been made: on one hand, some insist on a direct ontological interpretation of the wave function as representing physical bodies, on the other hand are those who claim that quantum mechanics is not really about the wave function.

In this chapter I will present and discuss one proposal of the latter kind that focuses on the notion of primitive ontology.

\section{Wave Function Ontology}

There is a realist take on quantum theories according to which quantum mechanics is a theory about the behavior of an object called the wave function. ${ }^{1}$ That is, the wave function mathematically represents a real, physical field that constitutes physical objects. For this reason, such a view has been called wave function ontology. One of the strongest arguments for this view is an argument by analogy. If in a physical theory there is a fundamental equation for the evolution of a given mathematical object, generally we feel justified to take this entity to represent physical objects. Consider classical mechanics: the fundamental equation of this theory is Newton's equation that describes the temporal

\footnotetext{
${ }^{1}$ See Albert 1992, 1996; Albert and Loewer 1996; Lewis 2004, 2005, 2006; Wallace 2002, 2003 and references therein.
} 
evolution of a point in three-dimensional space. It is natural to interpret such object as describing a particle whose dimensions are negligible, and this is exactly the way we take it: we conclude that classical mechanics is a theory that describes the behavior of point-particles. By analogy, we should do the same in quantum mechanics: given that in this theory there is a fundamental equation, Schrödinger's equation, for the temporal evolution of the wave function, we are entitled to take the wave function to represent physical objects as well.

As a consequence of this view, physical space is not the traditional threedimensional space. Rather, it is the space on which the wave function is defined: this is called "configuration space." Historically, configuration space has been introduced in classical mechanics for mathematical purposes. It is constructed from three-dimensional physical space: if there are $\mathrm{N}$ point-particles, each with position $r_{i}$ in three-dimensional space, then configuration space is defined as the space of the configurations of all particles. That is, an element $q$ of configuration space is given by $q=\left(r_{1}, r_{2}, \ldots, r_{\mathrm{N}}\right)$. As a consequence, if there are $\mathrm{N}$ particles in the universe, configuration space has dimension $M=3 \mathrm{~N}$. Observe that if one maintains that physical bodies are represented by the wave function, then literally there are no particles, and therefore there is no real reason to call such space "configuration space." The proponents of this view realize this, but the name sticks nonetheless.

Because the proposal is to take the wave function to represent physical objects, it seems natural to take configuration space as the true physical space. But clearly, we do not seem to live in configuration space. Rather, it seems obvious to us that we live in three-dimensions. Therefore, a proponent of this view has to provide an account of why it seems as if we live in a three-dimensional space even though we do not. Connected to that problem, we should explain how to "recover the appearances" of macroscopic objects in terms of the wave function. Using Wilfrid Sellars's terminology (Sellars 1962), we need to reconcile the scientific image (the image of the world that our best scientific theories are giving us) with the manifest image (the image of the world that we ordinarily experience).This is something that proponents of this view are working on. Whether this project is succeeding, and whether it is in principle possible have been challenged elsewhere. ${ }^{2}$

\section{Primitive Ontology}

There are people who find the view just presented unsatisfactory, and put forward different alternatives. In this chapter I focus on the proposal that

\footnotetext{
${ }^{2}$ See Monton 2002, 2006; and Allori forthcoming.
} 
involves the notion of primitive ontology. Other positions that do not explicitly refer to such notion have been proposed, ${ }^{3}$ but they are not discussed here.

The notion of primitive ontology was first proposed in Dürr et al. 1992 and Goldstein 1998, and then discussed in a little more details in Allori et al. 2008. The main idea is that all fundamental physical theories, from classical mechanics to quantum theories, share the following common structure:

- Any fundamental physical theory is supposed to account for the world around us (the manifest image), which appears to be constituted by threedimensional macroscopic objects with definite properties.

- To accomplish that, the theory will be about a given primitive ontology: entities living in three-dimensional space or in space-time. They are the fundamental building blocks of everything else, and their histories through time provide a picture of the world according to the theory (the scientific image).

- The formalism of the theory contains primitive variables to describe the primitive ontology, and nonprimitive variables necessary to mathematically implement how the primitive variables will evolve in time. $^{4}$

- Once these ingredients are provided, all the properties of macroscopic objects of our everyday life follow from a clear explanatory scheme in terms of the primitive ontology.

Several questions come to mind at this point -- from clarifications about the notion of primitive ontology to questions about the motivations to endorse this view instead of the wave function ontology. I do not directly compare the two approaches here, because this has been done elsewhere (Allori forthcoming). Rather, I present the primitive ontology idea and its framework per se, in particular analyzing the many roles (metaphysical, epistemological, and explanatory) that the primitive ontology has in the scientific enterprise. In doing so, some other questions will receive an answer:

- What is the primitive ontology for, and why is there an emphasis on its three-dimensionality (section 3)?

- Is there a rule to identify the primitive ontology of a theory (section 4)?

- What are the differences between the primitive and the nonprimitive variables (section 5)?

\footnotetext{
${ }^{3}$ See Monton 2002, 2006; Maudlin 2007a.

${ }^{4}$ The metaphysical status of such nonprimitive variables is up for debate (see section 8 , for instance), but surely they do not represent physical objects.
} 
- What is the connection between the primitive ontology and the explanatory power of a theory (section 6)?

- How can we apply the primitive ontology framework to quantum theories (section 7$)$ ?

- What is the status of the nonprimitive variables (in particular the wave function) in this picture (section 8)?

- What is the connection between symmetry properties of a theory and its primitive ontology (section 9)?

\section{Scientific and Manifest Image}

The primitive ontology proposal is tightly connected to a particular understanding of what physics is, what it does, and how it does it. In other words, it is connected to a particular understanding of what the scientific image is, how we arrive at it, and how it relates to the manifest image. The starting idea is that when a scientist proposes a fundamental physical theory, she already has in mind what the theory is fundamentally about: the primitive ontology. ${ }^{5}$ This is the metaphysical role of the primitive ontology: it tells us what the world is made of according to the theory.

But how does the scientist choose the primitive ontology? How do we go from the manifest to the scientific image? Or, how do we change from one old scientific image to a new one? To simplify and cut a long story short, in the words of Albert Einstein 1936, "the whole of science is nothing more than a refinement of everyday thinking." The scientific image typically starts close to the manifest image, gradually departing from it if not successful to adequately reproduce the experimental findings. The scientific image is not necessarily close to the manifest image, because with gradual departure after gradual departure we can get pretty far away. In fact, historically we went from the manifest image of a table being continuous and solid, to the Newtonian scientific image of the table being composed of microscopic particles and mostly empty. The point, though, is that the scientist will typically tend do make minimal and not very radical changes to a previously accepted theoretical framework. First, she might attempt to keep the same primitive ontology as the old theory, perhaps changing the law with which it evolves. If that fails, she might go for a different primitive ontology that still will not radically change her ways of understanding things. In other circumstances, she might move to a theory that will provide a better explanation.

\footnotetext{
${ }^{5}$ The qualification "fundamentally" is connected with the role of the primitive ontology in the structure of the theory, as we will see in section 6 .
} 
The situation is complex, and a lengthier discussion should perhaps be required, but let me clarify the main idea with a concrete example. At some point scientists attempted to explain thermal phenomena, such as two bodies in contact reaching the same temperature, positing a primitive ontology not too distant from the manifest image: heat was postulated to be a thermal fluid that passed from one body to the next. When this hypothesis did not work, a less "manifest" primitive ontology was proposed: each body was considered to be composed of many microscopic particles, and heat was understood as the motion of such particles. This is essentially Boltzmann's approach to thermodynamics, in which thermal phenomena are recovered from a Newtonian picture of the world when there is a very large number of particles. ${ }^{6}$ The particle primitive ontology was arguably suggested by the experimental failure of the more straightforward choice, the thermal fluid primitive ontology. At the same time, though, a primitive ontology of particles was not a radical choice, given that it was the primitive ontology of Newtonian mechanics, an already successful theory in other domains. Hence, roughly, in this sense the scientific image departed gradually from the manifest image to cope with the experimental results.

Through history, the primitive ontologies of the various fundamental physical theories have changed a lot: people have considered the world as made of fields, particles, flashes, ${ }^{7}$ strings, and so on. These proposed primitive ontologies have something in common: they all are in three-dimensional space, or in spacetime. ${ }^{8}$ Why is that? Because although it seems reasonable that we might be mistaken about what kind of entities the world is made of (fields, particles, and so on), to give up the idea that matter lives in three-dimensional space and evolve in time seems too much, especially if there is no need for it. A primitive ontology in the familiar three-dimensional space evolving in time (or a space-time primitive ontology) is the natural metaphysical choice, if the theory with such a primitive ontology can be empirically and explanatory adequate (namely, a good compromise between getting the empirical predictions right, and providing a satisfactory explanation of the phenomena). As in classical mechanics, it seems most convenient to explain, if possible, the behavior of familiar macroscopic bodies postulating that they are composed of microscopic entities in threedimensional space that constitute the fundamental building blocks of everything else. In fact, as we will see later, we can employ a clear scheme, developed in the framework of classical theories, to explain the properties of three-dimensional macroscopic objects in terms of the properties of their three-dimensional

\footnotetext{
${ }^{6}$ For a discussion, see Goldstein 2001 and Albert 2000.

${ }^{7}$ See section 7 .

${ }^{8}$ Even if the space of string theory is t10-dimensional, because all dimensions but three--are wrapped up on themselves ("compactified"), physical space is, for all practical purposes, threedimensional.
} 
microscopic constituents. We will also see how the same primitive ontology framework can be extended to quantum theories. Because of this, also in quantum theories we can account, at least in principle, for the macroscopic world along the lines of classical mechanics.

The primitive ontology provides us with a clear metaphysical picture of the world. So does the wave function ontology: the world is made of stuff represented by the wave function. One difference between the approaches is that the primitive ontology is in three-dimensional space (or in space-time), whereas the wave function is not. As a consequence of this, in the case of the wave function ontology, the scientific image does not have much in common with the previously accepted Newtonian picture. This is not true in the case of theories with a primitive ontology. In contrast to the case of wave function ontology, the primitive ontology approach reflects the desire to keep the scientific image closer to the classical way of understanding things, given that it is possible. The reason for this attitude, as we just saw, is obvious: if you can account for everything that you need to account for employing already successful and well-tested explanatory techniques, why not do so?

Why the qualification "primitive ontology," instead of just "ontology" simpliciter? First, the idea is that the primitive ontology does not exhaust all the ontology -- it just accounts for physical objects. Other things might exist (numbers, mathematical objects, abstract entities, laws of nature, and so on), and some of them (like natural laws) might be described by other objects in the ontology of a fundamental physical theory. We will see in section 8 how this could be true for the wave function. For more on the qualification "primitive," see section 6 . Now we turn to the general structure of fundamental physical theories in the primitive ontology framework.

\section{The Structure of Fundamental Physical Theories}

Assume that the idea of scientific image just discussed is correct. Hence, the scientist formulating a given theory will make a metaphysical hypothesis and develop her theory around it. Physics works through mathematics: a theory contains several mathematical objects, some with a physical significance, others without. The point here is that this is established once the theory is proposed: there is already a natural interpretation for each mathematical object, namely the one the proponent of the theory intended to give them! The scientist's choice of what physically exists in the world will more or less automatically determine the mathematical object to represent it. A fundamental physical theory aims to describe not only what physical bodies there are, but also how they evolve in time. Because of this, in addition to the variables describing the primitive 
ontology, the theory also contains some other equations, whose solutions describe how the primitive ontology moves through space in time.

The mathematical formalism of a theory therefore has a history that constrains the interpretation of its formalism: the theory started with a metaphysical position and its appropriate mathematical representation, and it continued with the implementation of the suitable mathematical apparatus necessary to determine how the primitive ontology evolves. For this reason, the argument by analogy already discussed for the wave function ontology view is misguided: it assumes in fact that the mathematical formalism of a theory can be interpreted a posteriori, whereas it was fixed a priori by the physicist when she formulated the theory. Therefore, there is no rule to determine the primitive ontology of a theory. Instead, it is a matter of understanding how the theory was introduced, how it has developed, and how its explanatory scheme works (for this, see section 6). Once the scientist sets up the theory, the metaphysical picture it provides has already been defined, and there is very limited freedom of reinterpreting the formalism (at least with the limitations exemplified by the case of classical electrodynamics, as we will see shortly).

Let us explicitly see how this framework works with the aid of some examples. As anticipated, a clear case of a fundamental physical theory with a primitive ontology is classical mechanics. In this theory physical objects are taken to be particles, and Newton's equation captures the temporal evolution of these objects via the introduction of forces and masses. These are "additional" variables in classical mechanics in the sense that they were added into the theory to account for the behavior of the primitive ontology.

Another example is given by classical electrodynamics (CED). The theory was developed initially from classical mechanics to account for the evolution of charged particles. New mathematical entities were introduced - the electromagnetic fields. Are the fields part of the primitive ontology of this theory? Actually, we can have different answers. On one hand, we can insist that the fields were added in the theory to account for the experimental trajectories of charged particles. If so, we have a theory, call it CEDp, in which fields do not represent matter, which is made only of particles. On the other hand, we might be inclined to think that the previous primitive ontology of particles was incomplete, and the fields indeed represent something in the material world. In this case we have a different theory, CEDpf, with a primitive ontology of particles and fields. In other words, the variables describing the electromagnetic fields can be regarded as nonprimitive, in the sense that their role in the theory is not to describe physical bodies but to implement the empirically correct behavior of physical bodies ([Dürr et al. 1992]). Instead other considerations (such as the fact that the electromagnetic fields have their own law of temporal evolution and 
there is energy associated with them) have led others to think that the electromagnetic fields represent part of the basic furniture of physical reality (see, for instance, Maudlin 2007a).

Be that as it may, to sum up, all these theories have a dual structure: the primitive variables that specify what matter is, and some other variables that determine its temporal development (its dynamics).

\section{Primitive and Nonprimitive Variables}

The histories of the primitive ontology - that is, their evolution in space through time, -provide the metaphysical picture of the world, and they are produced with the aid of (some of the) non-primitive variables. Just like a computer program generates an output, the fundamental physical theory "generates" the histories of the primitive ontology. And as the computer program needs certain internal variables to produce its output, the theory needs additional variables to implement the law of motion for the primitive ontology. Note that we could use different internal variables to obtain the same histories for the primitive ontology. If we do so, we still have \{fundamentally\} the very same theory: two theories with the same histories of the primitive ontology can be regarded as physically equivalent, because they provide us with the very same picture of the world.

This notion of physical equivalence between theories was introduced in Allori et al. 2008 in the framework of quantum mechanics. Nonetheless, it is not necessary to go to quantum theories to give an example of physically equivalent theories. Here is a very simple example of physical equivalent theories. If a force is conservative, it can be defined as the opposite of the gradient of the potential. This particular mathematical operation involves derivatives, and because of this it is always possible to find two different potentials that give rise to the same histories of the primitive ontology: any two potentials that differ by a constant will do the trick. In fact, they both give rise to the same force (and therefore the same histories of the primitive ontology), given that the derivative of any constant is always zero. Hence, two theories with such potentials will be physically equivalent

To conclude, two different theories with the same histories of the primitive ontology, no matter how they are implemented, describe the same physical world. The rest are details: how the dynamics for the primitive ontology is implemented is not important in this regard. This stresses the epistemological role of the primitive ontology: we only need to know its histories to recover the empirical data, given that the same histories could have been produced by different mathematical variables, as the previous example showed. 


\section{The Explanatory Scheme of Fundamental Physical Theories}

A microscopic primitive ontology grounds a scheme of explanation that allows one to determine the properties of macroscopic physical objects in terms of the behavior of the primitive ontology. In fact, in classical mechanics any physical body (gases, fluids, and solids) is satisfactorily described as a collection of particles. The story the theory tells us about the macroscopic world is a "geometrical story" - a table is just a table-shaped cluster of microscopic primitive ontology. Once the primitive ontology and its temporal evolution are given, everything else follows: the solidity of a table, the localization of a comet, the transparency of a pair of glasses, the liquidity of the water in a bottle, the compressibility of the air in a room, and so on. Arguably, in classical mechanics (as well as in classical electrodynamics) we can identify macroscopic properties more or less straightforwardly given how the microscopic primitive ontology combines and interacts to form complex bodies. ${ }^{9}$

Let us see how that works by way of some examples. First, we can explain why a table is solid on the basis of the fact that it is composed of particles that interact electromagnetically such that it is impossible for another object (for instance my hand) to penetrate them. Next, suppose we want to account for the fact that a comet has a given localization at a given time. One can accomplish this in terms of the microscopic components of the comet and their interaction with each other: the particles interact to form a solid object whose motion (and therefore its localization at different temporal instants) can be just as effectively described by its center of mass. Also, the transparency of an object such as a pair of glasses can be explained in terms of the electromagnetic forces acting between the particles composing the glasses, which are such that incoming light rays will pass through them. Similarly for fluids: a property like the liquidity of water can be explained in terms of the very weak interaction between the microscopic constituents of water that allow it to take the shape of its container. In addition, the behavior of gases is accounted for by considering them as composed by noninteracting particles colliding with one another. This is what happens when we derive thermodynamics from statistical mechanics: what in thermodynamics we call pressure, volume, temperature of a gas are derived from the fact that gases are composed of moving particles. Given that air is a gas, and given that a gas is just a collection of non-interacting particles, we can also explain why air is compressible: it is possible to reduce the distance between the particles almost as much as we want.

\footnotetext{
${ }^{9}$ An antireductionist would object to this, but granting that reductionism is possible, this is how it is supposed to work.
} 
These examples show how we have a clear and straightforward scheme of explanation in the classical framework: given the primitive ontology at the microscopic level, one can employ standard methods to determine the properties of familiar macroscopic objects. This is possible because classical theories have a primitive ontology, so for any other fundamental physical theory with a primitive ontology we could employ an explanatory scheme developed along the same lines.

Thus in this sense the primitive ontology is the most fundamental ingredient of the theory. It grounds the "architecture" of the theory: first we describe matter through the primitive variables, then we describe its dynamics, implemented by some nonprimitive variables, and that's it. All the macroscopic properties are recoverable. This summarizes the explanatory role of the primitive ontology. This is also connected with the "primitiveness" of the primitive ontology: even if the primitive ontology does not exhaust all the ontology, it makes direct contact between the manifest and the scientific image. Because the primitive ontology describes matter in the theory (the scientific image), we can directly compare its macroscopic behavior to the behavior of matter in the world of our everyday experience (the manifest image). Not so for the other nonprimitive variables, which can only be compared indirectly in terms of the ways they affect the behavior of the primitive ontology.

\section{Quantum Mechanics with Primitive Ontology}

Classical mechanics and classical electrodynamics provide two paradigmatic examples of how physics tells us about the world: in the scientific image there are the primitive variables that describe matter microscopically, and the manifest image, in which there are macroscopic objects with their properties, is obtained considering the histories of the primitive ontology in the appropriate macroscopic limit. It is a very nice explanatory scheme, straightforward and clear. Too bad it seems we have to abandon it once we consider quantum mechanics. In fact, several extremely strong assertions have been made about quantum theories - from the claim it is impossible to be realist if quantum mechanics is true, to the idea that the act of observation can affect reality, to the insistence that the "old," classical way of understanding the world we just described is no longer suitable.

The reasons for these attitudes can be perhaps understood by briefly recalling the history of quantum mechanics. At the end of the nineteenth century, the Newtonian picture of the world was commonly accepted, even if there were several puzzles: there were experiments whose results did not come out as the theory predicted. Some of them suggested the idea of quantization - a discretization of the values certain physical quantities can assume that does not substantially challenge the classical hypothesis that physical objects are made of 
particles. Other results suggested instead a change in the ontology: some experiments were taken to show that particles sometimes behave like waves. But particles and waves are incompatible ontologies! $!^{10}$ This wave-particle duality seemed crazy, and people tried to get around it. Louis de Broglie introduced a particular wave - the wave function - to account for the behavior of particles. He proposed to associate such wave to each particle as a "guide field" (deBroglie 1928), and Erwin Schrödinger later described the evolution of the wave function by his famous equation. De Brogie's idea was abandoned (perhaps too quickly) on the basis of some criticism by Wolfgang Pauli at the 1928 Solvay Congress. In addition, some other results (such as Heisenberg's uncertainty principle and von Neumann's theorem [von Neumann 1932]) were taken to show that quantum theories had to be about the wave function, not about particles. A further problem, however, was that the attempt to interpret quantum mechanics in a realist fashion as a theory about the wave function seemed to fail. In fact, when Schrödinger tried to do so, he discovered the so-called measurement problem (Schrödinger 1935): if the wave function completely describes physical systems, and it evolves according to the Schrödinger equation, then impossible macroscopic superpositions that we clearly never observe (such as the superposition of a living and a dead cat) are produced. Some proposed to solve this problem by introducing the observer actively into the theory: conscious observations "collapse" the wave function to one of the terms of the superposition. There are many reasons to consider this approach unsatisfactory, first because of the unfortunate reference to the observer in the formalization of the theory. ${ }^{11}$ Be that as it may, the result was that for nearly 20 years everyone gave up on any realistic interpretation of quantum mechanics.

Eventually, in the 1950s new and less problematic proposals to solve the measurement problems were made. Einstein did not like the status of quantum mechanics and proposed an argument to show that the formulation of quantum theory was incomplete and should be supplemented by "hidden variables" (Einstein Podolsky Rosen 1935). Einstein's attempt was unsuccessful, but David Bohm (1952), perhaps with a similar idea in mind, revised and updated de Brogie's particle-wave theory and showed that his theory solves the measurement problem. In Bohm's theory the description of any physical system is provided by the wave function supplemented by other variables, the particles' positions. In this way, the symmetry among the various terms of the superpositions (dead and living cat) is broken by the presence of the particle trajectories, and the

${ }^{10}$ Particles have definite positions in time, and their temporal evolution is represented by a trajectory in space-time; in contrast, waves are delocalized, spread-out objects that can diffract and interfere with one another.

${ }^{11}$ See Bell 1987, Maudlin 1995, and Goldstein 1998 among others. 
measurement problem is resolved - the cat is dead if the trajectories of the particles composing the cat fall in the support of the dead-cat wave function; she is alive if they fall in the support of the living-cat wave function. However, this theory had an unfortunate fate, since von Neumann's theorem was already taken to prove that hidden variables are impossible. This conviction was reinforced by certain presentations of Bell's inequality, developed in Bell 1964. As a result, Bohm's theory was dismissed for a long time: people believed there was something wrong with it, even if it was not clear what. Only fairly recently was it appreciated that the interpretations of these results were mistaken: it is possible for the quantum world to be made of particles, and there is nothing wrong with Bohm's theory. ${ }^{12}$ Still, only few scholars took the theory seriously, and some of them developed a better formulation that now goes under the name of Bohmian mechanics. ${ }^{13}$ Even if there are particles in Bohmian mechanics, people still insisted on the wave function. In fact, the other solutions to the measurement problem focused on either accepting the macroscopic superpositions, or eliminating them. Hugh Everett (1957) developed the so called many-worlds interpretation of quantum mechanics, in which the terms of the superpositions are interpreted as belonging to different worlds to which we have no access, so that everything that can happen (all superpositions) will happen, but in a different world. ${ }^{14}$ Another possible response to the measurement problem is the GRW theory, proposed by Ghirardi Rimini Weber (1986). In the GRW theory the wave function randomly collapses in one of the terms of the superpositions not because of an observer but as a result of a physical law: the wave function evolves according to a stochastic equation that allows for random spontaneous collapses. $^{15}$

These three examples show how it is possible to provide realist interpretations of the quantum formalism that do not rely on the notion of the observer. For this reason they have been called quantum theories without observers (Popper 1967, Goldstein 1998). Arguing along the lines of the ideas presented in section 1 , all these theories were naturally taken to be theories about the wave function, including Bohmian mechanics which was considered a theory about both the wave function and the particles. However, the concern with these theories is that, because the wave function lives on configuration space and not

\footnotetext{
${ }^{12}$ For a correct presentation of Bell's theorem see directly Bell 1964 or Dürr et al. 2004, where also the so-called no-go theorems against hidden variables theories are discussed.

${ }^{13}$ See, for example, Dürr et al. 1992, Allori and Zanghì 2004, and Goldsten 2001 for a review of Bohmian mechanics.

${ }^{14}$ For more on the many-worlds theory, see among others Vaidman 2002, Wallace 2002, Barrett 1998.

${ }^{15}$ For a review of the GRW theory, also called "spontaneous collapse theory," see for instance, Bassi and Ghirardi 2003 and Ghirardi 2002.
} 
three-dimensional space, the explanatory scheme developed in classical theories in terms of a primitive ontology must be drastically revised. A new explanatory scheme is needed, and nobody has found one yet. Hence, Bohmian mechanics, GRW and many-worlds, as theories of the wave function, at present, are not satisfactory theories (see Allori forthcoming).

We can avoid this problem by developing quantum theories with a primitive ontology. Various proposals have been made: they are quantum theories in which, as in classical theories, there is stuff in space-time, and we can develop a clear explanatory scheme, along the lines of the classical one, to account for the macroscopic world. As a consequence, there is no quantum revolution (or at least, not the one advertised so far): the quantum world is less crazy and paradoxical than one would have thought. This could be a disappointment for some, but certainly it is a great relief for others - we can still understand things the way we did before! To see where these proposals come from, let us go back to Bohmian mechanics. As we saw, one could think of it as a theory about both particles and the wave function, but if we look closely at its structure we see that this approach is contrived. In fact Bohmian mechanics is naturally a theory with a primitive ontology: there are particles (the primitive ontology), whose temporal evolution is governed by a Schrödinger evolving wave function (the nonprimitive variable). Having understood the role of the wave function in Bohmian mechanics, one can start to look differently to the other quantum theories without observers. The GRW theory as we described it, in which the Schrödinger evolution of the wave function is interrupted by random collapses, does not have a primitive ontology. But two distinct GRW-type theories with primitive ontology have been proposed, originally by Benatti et al. 1995 and Bell 1987 respectively: GRWm, a theory in which the primitive ontology is a field in three-dimensional space defined in terms of the wave function, representing the matter density of physical systems, and GRWf, a theory in which the primitive ontology is a set of discrete points in space-time called "flashes," whose rate depends on the wave function. ${ }^{16}$ In this case the primitive ontology is already in space-time, so the set of flashes already provides the set of histories of the primitive ontology. In both GRWm and GRWf the evolution of the primitive variables is determined by the wave function, which in turns evolves according to the modified GRW dynamics. In addition, Allori et al. 2011 have proposed and developed a many-worlds theory with primitive ontology that they called Sm: a matter density field ontology in three-dimensional space as in GRWm, combined with a Schrödinger evolving wave function that determines the temporal evolution of the primitive variables.

\footnotetext{
${ }^{16}$ For more on these theories, see for example Tumulka 2006, and Allori et al. 2008.
} 
A nonexhaustive list of other possible quantum primitive ontologies and their evolutions can be found in Allori et al. 2008.

Because in this framework quantum theories have the same structure as classical theories, in these theories we should be able to recover, at least in principle, all the macroscopic properties of physical objects using an explanatory scheme derived along the lines of the classical one. Indeed, this has been done for Bohmian mechanics in Allori et al. 2002 and Dürr et al. 2004. In the GRW and many-worlds frameworks, more work needs to be done. In any case, see Bassi and Ghirardi 2003 and Goldstein et al. 2011 for some related comments on the matter. ${ }^{17}$

\section{Wave Function and Primitive Ontology}

What about the wave function? In the primitive ontology framework, the wave function does not represent physical bodies. So what does it do? The role the wave function plays in the theory suggests how we should interpret it - in classical theories we needed other mathematical entities to implement the evolution for the particles, and here we need the wave function to implement the motion of the primitive variables. This is apparent in Bohmian mechanics, in which the wave function defines the evolution equation for the particles. In GRWm, GRWf and Sm the situation is analogous: the histories of the primitive variables are determined by the wave function. In Sm the wave function evolves according to Schrödinger equation as in Bohmian mechanics; in GRWf and GRWm it evolves stochastically. In addition, contrarily to Bohmian mechanics, in GRWf, GRWm and Sm, the wave function defines the primitive ontology. That is, in GRWm and in Sm the matter density is given by a certain function $m=m(\psi)$, and, analogously, in GRWf the set of flashes is determined by the wave function.

One could say that since in GRWm, Sm, and GRWf the matter density and the flashes supervene on the wave function, the wave function is "all that is needed," making the primitive ontology superfluous. But that would be a mistake: given a wave function $\psi$, different ways of defining the matter density and flashes are possible, even if only one is representing physical objects. To determine which one it is, we need more than just the wave function. Consider, for example, among the infinite possible ones, the following simple functions: $\mathrm{m}_{1}=\psi^{2}$ and $\mathrm{m}_{2}=\psi^{3}$. Both $\mathrm{m}_{1}$ and $\mathrm{m}_{2}$ are completely determined by $\psi$, but $\psi$ alone does not tell us which of

\footnotetext{
${ }^{17}$ Again, an antireductionist, would object to this, but the point here is that in quantum theories with a primitive ontology, we are not worse off than in classical mechanics. That is, whatever can be raised against reductionism in classical mechanics could also be raised here in principle. There is no additional problem for reductionism just due to the fact that we are in the quantum framework.
} 
the two really represents matter. This additional piece of information needs to be specified in addition to $\psi$. In other words, the primitive ontology naturally, not logically, supervenes on the wave function, given that there is a law specifying the connection between the primitive ontology and the wave function. Because of this, the information provided by the wave function alone is not enough.

So, the role of the wave functions in all these theories is to determine the law of motion for the primitive ontology. In this sense it has a law-like, nomological character. For this reason, Dürr et al. (1992) have proposed that the wave function should be intended as a physical law.

Objections have been raised to this interpretation of the wave function, most vividly by Brown and Wallace (2005). First, laws of nature are time-independent, whereas the wave function, in all quantum theories, evolves itself in time. Dürr et al. (1992) and Godlstein and Teufel (2001) have anticipated and replied to this objection claiming that, even if it might be difficult to accept the wave function as a law in the current theories, it will become straightforward once we reach a theory of quantum cosmology in which the wave function is static.

Another objection focuses on the fact that there seem to be multiple degrees of reality: there are material entities, the primitive ontology, and there are nomological entities, represented by the wave function. One could avoid the problem becoming a nominalist with respect to laws. As an alternative, one could maintain that laws exist as abstract entities. One could insist in fact that, even if the view has problems, they are not strong enough to make one abandon the view altogether (see Maudlin [2007b] for a recent realist proposal about laws of nature). Another possible option is to try to eliminate the wave function completely from the theory, as has been attempted by Dowker and Henson (2004), Dowker and Herbauts (2004), and Dowker and Herbauts (2005). ${ }^{18}$

Note that in classical electrodynamics the electromagnetic fields evolve in time according to Maxwell's equations. Thus, the situation of CEDpf (in which there are fields in the world, in addition to particles) seems very similar in this respect to quantum theories about the wave function: both the wave function and the fields represent matter, and both evolve in time. The difference is that the electromagnetic fields live on three-dimensional space, not configuration space. Hence, we could consider the fields as describing matter (together with particles) without departing too much from the manifest image, contrary to the situation in quantum mechanics. This is the reason a quantum theory about the

\footnotetext{
${ }^{18}$ Working with a particular GRW model on a lattice, they conjecture that the wave function can be eliminated as a necessary part of the theory. But in other places in their paper they seem to argue that it is not necessary to know the wave function to get the correct experimental predictions.
} 
wave function is less attractive than a classical electrodynamics about the electromagnetic fields.

\section{Symmetry Properties}

Before concluding I wish to add a quick remark about the importance of the primitive ontology in connection with the symmetry properties of a theory. Roughly put, a theory is said to be invariant under a given symmetry if the histories of the primitive ontology given by the theory, when transformed under the symmetry, will again be possible histories for the theory. ${ }^{19}$ That is, if the original and the transformed histories are both possible solutions of the equations of motions for the primitive ontology, the theory is invariant under that symmetry. The histories of the primitive ontology provide the metaphysical picture of the world, so if the theory is invariant under a given symmetry, this picture should not change under the symmetry transformation connected to the symmetry. Given their role, the nonprimitive variables will transform under the symmetry in such a way as to ensure that the histories of the primitive variables are invariant. In other words, becasue the histories of the primitive ontology need to remain invariant under the symmetry, and given that the evolution of the primitive ontology is determined by the wave function, the wave function will transform in a particular way to make this invariance happen.

Invariance is therefore a property of the dynamics of the primitive ontology: changing the primitive ontology of a theory might change its symmetry properties. So before asking whether a given theory has a given symmetry, it is necessary to identify its primitive ontology and see whether the transformed histories of the primitive ontology are still possible histories for the theory.

Particularly important for quantum mechanics is the question of relativistic invariance: it is usual to assume that a theory is relativistic-invariant if the law of evolution of the wave function is of a particular sort (whether it is a KleinGordon or a Dirac equation, for example). But that is a mistake, because the evolution of the wave function is not the thing to look at - whatever the evolution of the wave function is, what is important is the evolution of the primitive ontology. It is worthwhile to mention that the recognition of the importance of the primitive ontology has led to the construction of a relativistic invariant version of GRWf (Tumulka 2006), whereas GRWm still has no relativistic invariant formulation. Relativistic invariant single-particle extensions of Bohmian mechanics constructed more or less explicitly with a primitive ontology in mind have been proposed: in [Bohm and Hiley (1993) and Dürr et al. (1999)

${ }^{19}$ To be more precise, one should mention probability distributions as well. In this regard, see Allori et al. (2008). 
using a wave function evolving according to Dirac's equation, while in Berndl et al. (1996) and Nikolic (2005) the wave function used to implement the dynamic of the primitive ontology evolves according to the Klein-Gordon equation. ${ }^{20}$ Also, Allori et al. (2011) have developed relativistically invariant extensions of Sm.

\section{Conclusion}

I conclude summarizing the common structure of fundamental physical theories based on the notion of primitive ontology:

- Any fundamental physical theory contains a metaphysical hypothesis about what constitutes physical objects: the primitive ontology, which lives in three-dimensional space or space-time and constitutes the building blocks of everything else.

- In the formalism of the theory the variables representing the primitive ontology are called the primitive variables; in addition, there are other variables necessary to implement the dynamics for the primitive ontology: these variables could be interpreted as (part of) laws of nature.

- Once this is set, one can construct an explanatory scheme based on the one used in classical theories that allows one to determine, at least in principle, all the macroscopic properties of familiar physical objects in terms of the primitive ontology.

This structure holds for classical as well as for quantum theories. Thus, the power of the primitive ontology approach in quantum mechanics is the power of tradition of clear understanding, so to speak, given that in this framework many successful ingredients used in classical theories are preserved, such as the essence of its explanatory scheme. In this way, the quantum world ceases to be a mystery, and we can start doing metaphysics through physics as we did so far.

\footnotetext{
${ }^{20}$ These latter attempts have the peculiarity that they seem to involve a particle traveling back in time.
} 


\section{References}

- Albert, David Z., Quantum Mechanics and Experience. Cambridge, Mass: Harvard University Press (1992).

- Albert, David Z., Elementary Quantum Metaphysics. In: J. Cushing, A. Fine, and S. Goldstein (eds.), Bohmian Mechanics and Quantum Theory: An Appraisal. Boston Studies in the Philosophy of Science 184: 277-284 (1996).

- Albert, David Z., Time and Chance. Cambridge, Mass: Harvard University Press (2000).

- Albert, David. Z., and Barry Loewer, Tails of the Schrödinger's Cat. In: R. Clifton (ed.), Perspective on Quantum Realities: Non-relativistic, Relativistic, and Field Theoretic. Dordrecht: Kluwer, 81-91 (1996).

- Allori, Valia, Detlef Dürr, Sheldon Goldstein, and Nino Zanghì: Seven Steps toward the Classical World. Journal of Optics B 4: 482-488 (2002).

- Allori, Valia, The Metaphysics of Quantum Mechanics. In: S. Le Bihan (ed.), La philosopie de la physique: d'aujour'hui a demain. Paris: Edition Vuibert (forthcoming).

- Allori, Valia, Sheldon Goldstein, Roderich Tumulka and Nino Zanghì, On the Common Structure of Bohmian Mechanics and the Ghirardi-Rimini-Weber Theory. British Journal for the Philosophy of Science 59: 353-389 (2008).

- Allori, Valia, Sheldon Goldstein, Roderich Tumulka and Nino Zanghì, ManyWorlds and Schrödinger's First Quantum Theory. British Journal for the Philosophy of Science 62 (1): 1-27 (2011).

- Allori, Valia, and Nino Zanghì, What is Bohmian Mechanics. International Journal of Theoretical Physics 43: 1743-1755 (2004).

- Barrett, Jeff: Everett's Relative-State formulation of Quantum Mechanics. In: Stanford Encyclopedia of Philosophy (1998), available online at http://plato.stanford.edu..

- Bassi, Angelo, and GianCarlo Ghirardi, Dynamical Reduction Models. Physics Report 379: 257-426 (2003).

- Bell, John Stuart, On the Einstein Podolsky Rosen Paradox. Physics 1 (3): 195-200 (1964). Reprinted as chapter 2 of Speakable and Unspeakable in Quantum Mechanics\}, Cambridge: Cambridge University Press (1987).

- Bell, John Stuart, Speakable and Unspeakable in Quantum Mechanics. Cambridge: Cambridge University Press (1987).

- Benatti, Fabio, GianCarlo Ghirardi, and Renata Grassi, Describing the Macroscopic World: Closing the Circle within the Dynamical Reduction Program. Foundations of Physics 25: 5-38 (1995). 
- Berndl, Karin, Detlef Dürr, Sheldon Goldstein, and Nino Zanghì, Nonlocality, Lorentz Invariance, and Bohmian Quantum Theory. Physical Review A 53: 20622073 (1996).

- Bohm, David, A Suggested Interpretation of the Quantum Theory in Terms of "Hidden" Variables, I and II. Physical Review 85: 166-193 (1952).

- Bohm, David, and Basil J. Hiley, The Undivided Universe: An Ontological Interpretation of Quantum Theory. London: Routledge and Kegan Paul (1993).

- Brown, Harvey, and David Wallace, Solving the measurement problem: de Broglie-Bohm loses out to Everett. Foundations of Physics 35: 517-540 (2005).

- de Broglie, Louis, La nouvelle dynamique des quanta. In: Solvay Congress 1927, Electrons et Photons: Rapports et Discussions du Cinquieme Conseil de Physique tenu a Bruxelles du 24 au 29 Octobre 1927 sous les Auspices de l'Institut International de Physique Solvay. Paris: Gauthier-Villars (1928).

- Dowker, Fay, and Joe Henson, Spontaneous Collapse Models on a Lattice. Journal of Statistical Physics 115: 1327-39 (2004).

- Dowker, Fay, and Isabelle Herbauts, Simulating Causal Wave-Function Collapse Models. Classical and Quantum Gravity 21: 2963-2979 (2004).

- Dowker, Fay, and Isabelle Herbauts, The Status of the Wave Function in Dynamical Collapse Model. Foundations of Physics Letters 18: 499-518 (2005).

- Dürr, Detlef, Sheldon Goldstein, and Nino Zanghì, Quantum Equilibrium and the Origin of Absolute Uncertainty. Journal of Statistical Physics 67: 843-907 (1992).

- Dürr, Detlef, Sheldon Goldstein, Karin Münch-Berndl, and Nino Zanghì, Hypersurface Bohm-Dirac Models. Physical Review A 60: 2729-2736 (1999).

- Dürr, Detlef, Sheldon Goldstein, and Nino -Zanghì, Quantum Equilibrium and the Role of Operators as Observables in Quantum Theory. Journal of Statistical Physics 11: 959-1055 (2004).

- Einstein, Albert, Physics and Reality. Reprinted in: A. Einstein, Out of My Later Years. New York: Philosophical Library (1950).

- Einstein, Albert, Boris Podolsky, and Nathan Rosen, Can Quantum-Mechanical Description of Physical Reality be Considered Complete? Physical Review 47 (10): 777-780 (1935).

- Everett, Hugh, Relative State Formulation of Quantum Mechanics. Review of Modern Physics 29: 454-462 (1957).

- Ghirardi, GianCarlo, Collapse Theories. On: Stanford Encyclopedia of Philosophy (2002), available online at http://plato.stanford.edu.

- Ghirardi, GianCarlo, Alberto Rimini, and Tulio Weber: Unified Dynamics for Microscopic and Macroscopic Systems. Physical Review D 34: 470-491 (1986).

- Goldstein, Sheldon, Quantum Theories without Observers. Physics Today 51, 3: 4246, and 4: 38-42 (1998). 
- Goldstein, Sheldon, Boltzmann's Approach to Statistical Mechanics. In: J. Bricmont, D. Dürr, M. C. Galavotti, G. C. Ghirardi, F. Petruccione, and N. Zanghì (eds.), Chance in Physics: Foundations and Perspectives. New York: Springer-Verlag, (2001).

- Goldstein, Sheldon, Bohmian Mechanics. In: Stanford Encyclopedia of Philosophy (2006), available online at http://plato.stanford.edu.

- Goldstein, Sheldon, and Stefan Teufel, Quantum Spacetime without Observers: Ontological Clarity and the Conceptual Foundations of Quantum Gravity. In: C. Callender and N. Huggett (eds.) Physics meets Philosophy at the Planck Scale. Cambridge University Press : 275-289 (2001).

- Goldstein, Sheldon, Roderick Tumulka, and Nino Zanghì, The Quantum Formalism and the GRW Formalism (2011). Available online at http://arxiv.org/pdf/0710.0885.pdf.

- Lewis, Peter, Life in Configuration Space. British Journal for the Philosophy of Science 55: 713-729 (2004).

- Lewis, Peter, Interpreting Spontaneous Collapse Theories. Studies in History and Philosophy of Modern Physics 36\}: 165-180 (2005).

- Lewis, Peter, GRW: A Case Study in Quantum Ontology. Philosophy Compass l: 224-244 (2006).

- [Maudlin, Tim, Three Measurement Problems. Topoi 14: 7-15 (1995).

- Maudlin, Tim, Completeness, Supervenience and Ontology. Journal of Physics A: Mathematical and Theoretical 40: 3151-3171 (2007).

- Maudlin, Tim, The Metaphysics within Physics. Oxford: Oxford University Press (2007).

- Monton, Bradley, Wave Function Ontology. Synthese 130: 265-277 (2002).

- Monton, Bradley, Quantum Mechanics and 3N-Dimensional Space. Philosophy of Science 73: 778-789 (2006).

- Nikolic, Hrvoje, Relativistic Quantum Mechanics and the Bohmian Interpretation. Foundations of Physics Letters 18: 549-561 (2005).

- Popper, Karl, Quantum Theory without "the Observer." In: M. Bunge (ed.), Quantum Theory and Reality, Berlin: Springer (1967).

- Schrödinger, Erwin, Die gegenwärtige Situation in der Quantenmechanik. Naturwissenschaften 23: 807-812; 823-823, 844-849 (1935). English translation: John D. Trimmer, Proceedings of the American Philosophical Society 124: 323-338 (1980).

- Sellars, Wilfrid, Philosophy and the Scientific Image of Man. In: R. Colodny (ed.), Frontiers of Science and Philosophy, Pittsburgh: Pittsburgh University Press (1962). Reprinted in: Science, Perception and Reality, London: Routledge (1963).

- Tumulka, Roderich, A Relativistic Version of the Ghirardi-Rimini-Weber. Journal of Statistical Physics 125: 821-840 (2006). 
- Vaidman, Lev, Many-Worlds Interpretation of Quantum Mechanics. In: Stanford Encyclopedia of Philosophy (2002), available online at http://plato.stanford.edu.

- von Neumann, John, Mathematische Grundlagen der Quantenmechanik, Springer (1932). English translation: Mathematical Foundations of Quantum Mechanics, Princeton University Press (1955).

- Wallace, David, Worlds in the Everett Interpretation. Studies in the History and Philosophy of Modern Physics 33: 637-661 (2002).

- Wallace, David, Everett and Structure. Studies in the History and Philosophy of Modern Physics 34: 86-105 (2003). 